\title{
Les effets collatéraux du dépistage du cancer colorectal
}

\author{
Side Effects of Colorectal Cancer Screening
}

\author{
E. Vaillant \\ (C) Lavoisier SAS 2016
}

Un petit tour d'Europe du dépistage a permis de montrer une disparité d'incidence assez importante entre les pays du sud (Grèce, Chypre) mais aussi la Finlande et les pays du centre de l'Europe (Hongrie, Tchéquie, Slovaquie) où l'incidence est la plus forte, variant de 1 à 5 . Concernant la survie à cinq ans, la France est $7^{\mathrm{e}}$, principalement à cause des hommes qui ont une mortalité plus importante. Il ne reste finalement que deux méthodes utilisées : la recherche de sang dans le selles (FIT ou Hemoccult) et l'endoscopie (rectosigmoïdoscopie ou coloscopie) avec de grandes variations de stratégie selon les pays européens. Vingt-quatre des 28 pays de l'Union ont un programme de dépistage organisé ou opportuniste. Ce qui apparaît important est de savoir que la baisse de l'incidence du cancer colorectal que l'on peut espérer sera principalement liée au taux de participation dans les programmes de dépistage et qu'il n'y pas une méthode supérieure à l'autre en termes de coût-efficacité de manière évidente, les capacités de recours à l'endoscopie et les préférences de la population sont les deux points qui influent sur le choix entre les deux méthodes, une meilleure adhésion est obtenue lorsqu'on laisse le choix.

Dans une étude espagnole [1] portant sur une population ayant réalisé un FIT, une recherche de mutation APC ou MUTYH était effectuée chez ceux qui présentaient plus de dix adénomes. On retrouvait plus de dix adénomes dans $2,7 \%$ des coloscopies et dans $11 \%$ de ces cas, une mutation de polypose atténuée ou de forme sans doute très atténuée était retrouvée (APC ou MUTYH). Le dépistage permet donc aussi d'identifier des personnes à risque élevé ou très élevé qui relèvent d'une consultation d'oncogénétique, mais dont le suivi n'est pas encore codifié, et il sera sans doute nécessaire de préciser les modalités de surveillance par des études.

Les petites tumeurs neuroendocrines du rectum sont fréquentes et leur incidence augmente en raison d'un meilleur diagnostic endoscopique. Dans $80 \%$ des cas, ces tumeurs sont $<1 \mathrm{~cm}$ et n'ont pas de critère invasif. L'ENETS (Société européenne des tumeurs endocrines) précise dans ses guidelines de 2012 que les tumeurs $<2 \mathrm{~cm}$ peuvent être réséquées endoscopiquement). Dans une étude coréenne multicentrique portant sur 14 centres universitaires et sur dix ans, différentes techniques ont été comparées sur une vaste cohorte de 572 patients. Les techniques de polypectomie - mucosectomie (EMR) classique ou modifiée (par ligateur, avec cap ou précoupe) et dissection sous-muqueuse (ESD) étaient comparées en termes d'efficacité et de complication. Il s'agissait de tumeurs d'une taille de 5,7 $\mathrm{mm}$ en moyenne, l'analyse des marges de résection était négative dans $64 \%$ des cas et indéterminée dans $18 \%$. Les lésions étaient essentiellement sous-muqueuses, il n'y avait d'invasion lymphovasculaire que dans $1,7 \%$ des cas et le suivi médian était de 56 mois. Des marges saines étaient obtenues de manière significative avec l'ESD et l'EMR modifiée. Un saignement est survenu dans $13 \%$ des cas et une perforation dans $3 \%$. Les récidives étaient très peu fréquentes dans $0,74 \%$ des cas, que ce soit locales ou métachrones. Il n'y avait pas de différence significative en termes de complication selon les différentes méthodes. Cette étude valide le traitement endoscopique et recommande une surveillance endoscopique régulière pour les tumeurs $<10 \mathrm{mn}$.

\section{Références}

1. Ono A, Carbonell P, Alajarin M, et al. Prevalence of APC/MYH germline mutations detected on a population based colorectal cancer screening programme in Spain. 2015;Congrès UEGW ; OP 045.

2. Chang MM. Effectiveness and safety of endoscopic resection for rectal neuroendocrine tumors according to different resection methods: A Kasid multi-center study. 2015;Congrès UEGW ; OP 073.

E. Vaillant $(\bowtie)$

11033 , avenue de la République, F-59700 Marcq-en-Barœul

e-mail : vaillant-e@orange.fr 